University of Texas at El Paso

ScholarWorks@UTEP

$12-2016$

\title{
For Fuzzy Logic, Occam's Principle Explains the Ubiquity of the Golden Ratio and of the 80-20 Rule
}

Olga Kosheleva

The University of Texas at El Paso, olgak@utep.edu

Vladik Kreinovich

The University of Texas at El Paso, vladik@utep.edu

Follow this and additional works at: https://scholarworks.utep.edu/cs_techrep

Part of the Computer Sciences Commons

Comments:

Technical Report: UTEP-CS-16-98

Published in Journal of Innovative Technology and Education, 2017, Vol. 4, No. 1, pp. 13-18.

\section{Recommended Citation}

Kosheleva, Olga and Kreinovich, Vladik, "For Fuzzy Logic, Occam's Principle Explains the Ubiquity of the Golden Ratio and of the 80-20 Rule" (2016). Departmental Technical Reports (CS). 1081.

https://scholarworks.utep.edu/cs_techrep/1081

This Article is brought to you for free and open access by the Computer Science at ScholarWorks@UTEP. It has been accepted for inclusion in Departmental Technical Reports (CS) by an authorized administrator of ScholarWorks@UTEP. For more information, please contact Iweber@utep.edu. 


\title{
For Fuzzy Logic, Occam's Principle Explains the Ubiquity of the Golden Ratio and of the 80-20 Rule
}

\author{
Olga Kosheleva and Vladik Kreinovich \\ University of Texas at El Paso \\ $500 \mathrm{~W}$. University \\ El Paso, TX 79968, USA \\ olgak@utep.edu,vladik@utep.edu
}

\begin{abstract}
In this paper, we show that for fuzzy logic, the Occam's principle that we should always select the simplest possible explanation - explains the ubiquity of the golden ratio and of the 80-20 rule.
\end{abstract}

\section{From 2-Valued to Fuzzy Logic: Successes and Challenges}

Need for expert knowledge. In many real-life situations, from performing surgeries to piloting planes, we relay on experts' knowledge and skills.

Experts' skills differ: some experts are better, some are not as experienced. In the ideal world, it is desirable for everyone to use the experience of the best experts. However, in practice, there are a few best surgeons - and they cannot perform all the surgeries, there are a few best pilots - and they cannot pilot all the planes.

It is therefore desirable to incorporate the knowledge of the best experts into a computer-based system, so that other experts can use this knowledge.

Need for logic in the description of expert knowledge. Expert knowledge often comes in logical terms. For example, experts often formulate conditions for performing a certain action by saying that some condition should be satisfied and some other condition must be satisfied, etc. So, to describe such knowledge, we need to use "and"-operation (disjunction).

Sometime, conditions assume that some property is not satisfied. To describe such knowledge, we also need to use "not"-operation (negation).

Such "and"- and "not"-combinations are ubiquitous, e.g., in medicine, where usually, several conditions (some positive, some negative) are needed to make a diagnosis (and thus, to come up with an appropriate patient treatment). 
Traditional (2-valued) logic. If we have a statement $A$ with truth value $a \in\{0,1\}$ (0 means false, and 1 means true), and we can apply "and"-operation (\&) and negation $\neg$, then all we can get by applying these two operations (not counting trivially true and trivially false statements) are:

- the original statement $A$ with truth value $a$, and

- its negation $\neg A$ with truth value $1-a$.

Indeed, in classical logic, $S \& S$ is equivalent to $S$, and $S \& \neg S$ is always false.

Need for taking uncertainty into account. The traditional (2-valued) fuzzy logic assumes that for each of his/her statements, the expert is absolutely sure that this statement is absolutely true. In real life, experts are not absolutely sure about their statements. For example, a medical doctor can use a diagnostic rule that works in $99 \%$ of the cases, but he/she understands that there are exceptional cases when this rule is not applicable.

To adequately describe expert knowledge, it is therefore important to describe not only the expert's statements, but also their degrees of confidence in these statements. This is the main idea behind fuzzy logic (see, e.g., $[1,4,5]$ ), where, to each statement, we assign a number from the interval $[0,1]$ that describes to what extent the expert is confident in this statement: degree 1 means absolute confidence, degree 0 means that the expert is absolutely sure that the statement is false, and degrees between 0 and 1 correspond to different intermediate levels of confidence.

"And"- and "not"-operations in the presence of uncertainty: towards fuzzy logic. In the case of uncertainty, how can we interpret "and"- and "not"operations? This is a challenge, since while in the 2-valued logic, the truth values of the two statements $A$ and $B$ uniquely determine the truth value of $A \& B$, for intermediate degrees, knowing the degrees of confidence $a$ and $b$ in two statements does not allow us to uniquely determine the degree of confidence in $A \& B$.

For example, consider the case when $A$ is "a fair coin falls head", and let $a>0$ be the expert's degree of confidence that the coin will fall head. Since it is equally possible that a fair coin will fall head or fall tail, the expert's degree of confidence that the coin will fall tail is equal to the same number $b$.

So, if $B$ is "coin falls tail", then the expert's degrees of confidence $d(A)$ and $d(B)$ in $A$ and $B$ are the same: $d(A)=d(B)=a$. It is not possible for a coin to fall head and tail at the same time, so in this case, $d(A \& B)=0$.

On the other hand, if we take $B=A$, then $A \& B$ is simply equivalent to $A$, so in this case, we similarly have $d(A)=d(B)=a$ but this time, $d(A \& B)=$ $d(A)=a>0$.

Since the expert's degree of confidence in $A \& B$ is not uniquely determined by his/her degrees of confidence in $A$ and $B$, in the ideal world, we should elicit, from the expert, hsi/her degrees of confidence in all possible "and"-combinations of the original statements. However, if we have $n$ original statements, then we have $2^{n}$ possible "and"-combination. For reasonable size $n$, the number $2^{n}$ of 
such combinations becomes astronomical, exceeding the lifetime of the Universe. Thus, we cannot always elicit these degrees of confidence. We therefore need to estimate the degree of confidence $d(A \& B)$ based only on the known expert's degrees of confidence $a=d(A)$ and $b=d(B)$. The estimating degree of confidence is denoted by $f_{\&}(a, b)$, and the algorithm for computing this estimate is known as an "and"-operation or a $t$-norm.

Properties of "and"-operations (t-norms). The "and"-operation should satisfy some natural conditions. For example, since $A \& B$ and $B \& A$ mean the same, the estimates of the expert's degree of confidence for these two expressions should be the same, i.e., we should have $f_{\&}(a, b)=f_{\&}(b, a)$. In other words, the "and"-operation should be commutative.

Similarly, the fact that the "and"-combinations $A \&(B \& C)$ and $(A \& B) \& C$ are equivalent implies that we should have $f_{\&}\left(a, f_{\&}(b, c)\right)=$ $f_{\&}\left(f_{\&}(a, b), c\right)$, i.e., that the "and"-operation should be associative.

It is also reasonable to require that the "and"-operation is (non-strictly) monotonic in each of the variables, continuous, and satisfies the properties $f_{\&}(0, a)=0$ and $f_{\&}(1, a)=a$.

Which "and"-operations should we choose: let us use the Occam principle. There are many different "and"-operations that satisfy all these requirements. When selecting an operation, a reasonable idea is to use the Occam principle - i.e., to select the simplest possible "and"-operation.

Since our goal is to design a computer-based system, a reasonable way to measure simplicity vs. complexity is by comparing the computational complexity of different "and"-operations. From this viewpoint, the simplest case is to use functions $f_{\&}(a, b)$ which are the easiest to compute - i.e., which can be implemented by the smallest possible number of fast (hardware-supported) computer operations. In modern computers, operations which are hardware supported are - in the order of increasing computation time - min and max, addition and subtraction, multiplication, and division. Out of these operations, only two satisfy all the properties of the "and"-operations: min and product.

So, the simplest "and"-operation is $f_{\&}(a, b)=\min (a, b)$. This operation was indeed proposed as the main option in Zadeh's pioneer paper [5] that introduced fuzzy logic, and it remains one of the most widely used "and"-operations. However, it has a limitation: for this operation, $f_{\&}(a, a)=a$, so this operation does not take into account that if we use several not-fully-confident steps to reach a conclusion, our confidence in this conclusion becomes smaller and smaller as the number of steps decreases.

To avoid this limitation and make our treatment of degrees of confidence more adequate, it is therefore reasonable to consider the next simplest "and"operation: the algebraic product $f_{\&}(a, b)=a \cdot b$. The algebraic product has indeed been successfully used in many applications of fuzzy techniques.

Remaining challenge. Occam's principle helps us select an "and"-operation, but we still have a challenge of selecting appropriate real numbers to describe experts' degrees of confidence. 
What we do in this paper. In this paper, we show that the ideas behind Occam's principle can also help us select appropriate degrees of confidence.

Interesting, the resulting degrees seem to explain the ubiquity of the golden ratio and of the $80-20$ principle: that $20 \%$ of the people drink $80 \%$ of all the beer, that $20 \%$ of the people earn $80 \%$ of all the money, etc.

\section{How to Apply Occam's Principle to Select Ap- propriate Degrees of Confidence}

Main idea. We have a statement $A$, and we would like to assign a numerical degree describing the expert's confidence about this statement. To do it, let us consider not only the statement $A$ by itself, but also whatever auxiliary statements we can form from $A$. To form these auxiliary statements, we can use logical operations "and" and "not", and basic truth values.

Main idea in the 2-valued case. Let us start our analysis with the case of the two-valued logic.

In the 2-valued case, there are only two basic truth values: "true" (which is usually described by 1 ) and "false" (which is usually described by 0 ). In the 2 -valued logic, the "and"-operation does not change anything, so, in addition to 0 and 1 , we have only two possible statements: the statement $A$ itself and its negation $\neg A$ (and the result of using "and" to combine $A$ and $\neg A$ is just "false" - i.e., equal to 0$)$.

Extending our main from the 2-valued case to the general fuzzy case. In the fuzzy case, in addition to 0 and 1 , we also have additional (intermediate) truth values. The simplest case is when we consider one additional truth value - whose natural interpretation is "unknown". This additional truth value has to be equally separated from both "true" and "false", so it makes sense to take, as this value, a real number which is equidistant from 0 and 1 -i.e., the number 0.5 .

Let us now see what we can do if we also allow "and"- and "not"-operations. In principle, starting with the original statement $A$ with degree of certainty $a \in(0,1)$, we can have infinitely many different values $a^{2}$ (corresponding to $A \& A), a^{3}$ (corresponding to $A \& A \& A$ ), $a^{4}$ (corresponding to $A \& A \& A \& A$ ), etc.

Occam's principle - that we should create new entities unless it is absolutely necessary - seems to indicate that not all these statements should be different, some of them should coincide. Let us analyze the simplest cases of such coincidence.

Simplest case: when $A \& A$ has the same degree of confidence as some other auxiliary statement. Let us start with the case when the simplest possible "and"-combination $A \& A$ has the same degree of confidence as some other auxiliary statement. 
The simplest choice of such additional auxiliary statement - that does not require using any logical operation - is the value "unknown". The coincidence between the degree of confidence in $A \& A$ and the value "unknown" means that $a^{2}=0.5$. So, we arrive at the first reasonable degree of confidence:

$$
a=\sqrt{0.5}=\frac{\sqrt{2}}{2} \approx 0.7 .
$$

The next in complexity is the case when the additional statement requires a single logical operation. The only such case - in addition to the statement $A \& A$ itself - is the negation $\neg A$. So, we arrive at the case when the statements $A \& A$ and $\neg A$ have exactly the same degree of confidence: $a^{2}=1-a$. Interestingly, this is exactly the golden ratio

$$
a=\frac{\sqrt{5}-1}{2} \approx 0.68
$$

which is indeed ubiquitous in many practical applications.

Comment. It should be mentioned that the fuzzy origin of the golden ratio was first noticed in [3].

What next: when $A \& A \& A$ has the same degree of confidence as some other auxiliary statement. If the degree of confidence in a statement $A$ is different from $\sqrt{0.5}$ and from the golden ratio, then the "and"-combination $A \& A$ does not have the same degree of confidence as some other auxiliary statement. In this case, a natural way to describe Occam's principle is to require that the next "and"-combination $A \& A \& A$ has the same degree of confidence as some other auxiliary statement.

The simplest of all such cases if the case when the "and"-combination $A \& A \& A$ has the same degree of confidence as the simplest auxiliary statement - "unknown". This coincidence means that we have $a^{3}=0.5$. The corresponding value of $a$ - the cubic root of 0.5 - is very close to 0.8 (since $\left.0.8^{3}=0.512 \approx 0.5\right)$.

With the degree of $\neg A$ being $\approx 1-0.8=0.2$, this may explain the ubiquity of the 80-20 rule in many practical applications: that a few people drink most of the beer, that a few people earn most of the money, etc.; see, e.g., [2].

\section{Acknowledgments}

This work was supported by the National Science Foundation grants HRD0734825 and HRD-1242122 (Cyber-ShARE Center of Excellence) and DUE0926721, and by an award "UTEP and Prudential Actuarial Science Academy and Pipeline Initiative" from Prudential Foundation.

\section{References}

[1] G. Klir and B. Yuan, Fuzzy Sets and Fuzzy Logic, Prentice Hall, Upper Saddle River, New Jersey, 1995. 
[2] R. Koch, The 80/20 Principle and 92 Other Powerful Laws of Nature: The Science of Success, Nicholas Brealey, London, U.K., 2014.

[3] M. Koshelev, "Fuzzy logic explains the golden proportion", International Journal of Intelligent Systems, 1997, Vol. 12, No. 5, pp. 415-417

[4] H. T. Nguyen and E. A. Walker, A First Course in Fuzzy Logic, Chapman and Hall/CRC, Boca Raton, Florida, 2006.

[5] L. A. Zadeh, "Fuzzy sets", Information and Control, 1965, Vol. 8, pp. 338353. 